\title{
Congenital Torticollis with Bilateral Sternocleidomastoid Muscle Contracture
}

\author{
Siha Park', Soo A Kim' ', Jun Hwan Song' ${ }^{2}$, Ho Kim² \\ Departments of ${ }^{1}$ Physical Medicine and Rehabilitation and ${ }^{2}$ Pediatrics, Soonchunhyang University Cheonan Hospital, Soonchunhyang University College of \\ Medicine, Cheonan, Korea
}

\begin{abstract}
Congenital muscular torticollis is a common musculoskeletal disorder characterized by the shortening of the sternocleidomastoid muscle, thus leading to an ipsilateral tilt of the head and contralateral rotation of the face. Most of the congenital muscular torticollis is ipsilateral. Bilateral congenital muscular torticollis is extremely rare. Congenital muscular torticollis is known to be associated with several musculoskeletal and neurologic disorders including brachial plexus injury. Therefore, early diagnosis and intervention are crucial for the treatment and prevention of associated complications. Here, we report a case of a 1-month-old infant with bilateral congenital muscular torticollis suspicious of Erb-Duchenne palsy.
\end{abstract}

Keywords: Congenital torticollis; Brachial plexus injury; Physical therapy modalities

\section{INTRODUCTION}

Congenital muscular torticollis (CMT) is a common musculoskeletal problem in infants. The incidence rate of congenital muscular torticollis is $0.4 \%$, ranging from $0.3 \%$ to $1.9 \%$ [1] and characterized by fibrosis which results from shortening or contraction of sternocleidomastoid muscle (SCM) that leads to the ipsilateral tilt of the head and contralateral rotation of the face, chin, and facial asymmetry [2,3]. Congenital muscular torticollis is known to be associated with several problems including brachial plexus injury. Unilateral congenital torticollis is well known, whereas bilateral congenital muscular torticollis is a very rare form of the muscleskeletal disorder. We present a case of an infant with bilateral congenital torticollis with motor weakness in the right upper extremity.

\section{CASE REPORT}

The 1-month-old infant was referred to the rehabilitation department from the pediatric department during admission for systemic skin peeling right after birth. The patient's caregiver provided verbal informed consent for the publication of clinical details and images. She was the first baby from the pre-term cesarean section in a breech position. She was consulted for diagnosis of congenital muscular torticollis. The physical examination indicated asymmetry of the head and limited rotation of movement of the neck. The head presented an altered position of bending to the left side with chin rotated to right. Increased tension of both SCM restricted its movements and widened the shape of neck. Range of motion (ROM) in the neck was flexion $10^{\circ}$, extension $10^{\circ}$, lateral flexion right/left $15^{\circ} / 10^{\circ}$, and rotation right/left $70^{\circ} / 20^{\circ}$. She looked uncomfortable and expressed severe pain when rotating her neck to bilateral sides. For diagnosis, we performed an ultrasonographic study. The findings were hyperechoic lesions within the SCM on both sides (Fig. 1). The lesion ranged in size $30.9 \mathrm{~mm}$ and $29.9 \mathrm{~mm}$ on maximal transverse diameter, with length ranging 10.6-11.0 mm, on each side. Bilateral congenital torticollis was diagnosed. To rule out other pathologies, we performed X-ray, magnetic resonance imaging, ophthalmological, and otorhinolaryngological evaluation. All the results of the examinations were normal.

The infant showed prominent motor weakness on the right upper extremity (grade 1), specifically the arm could not be raised from the side, the elbow was extended and the forearm was pronated, which is suspicious of Erb-Duchenne palsy. We did not per- 

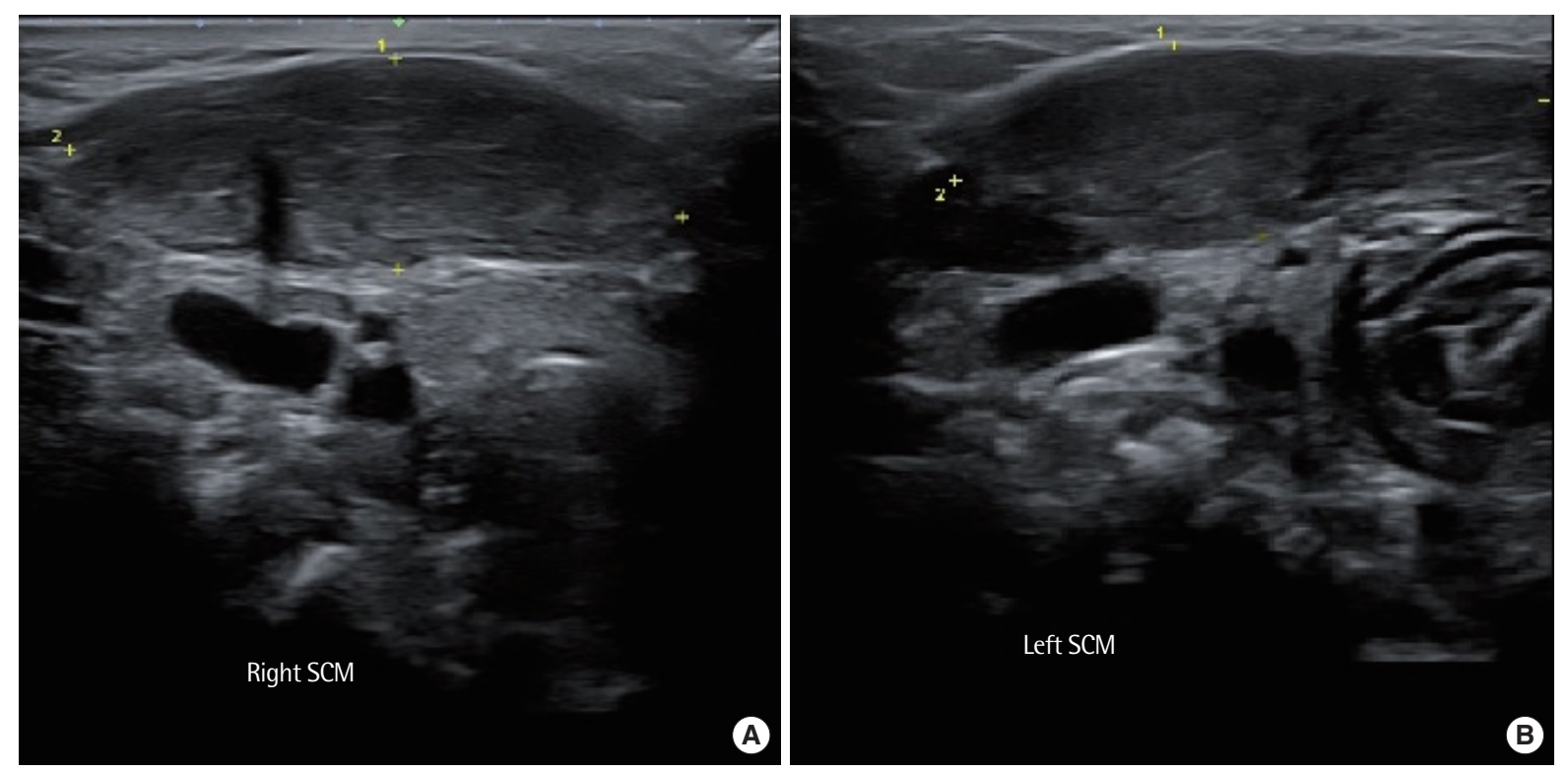

Fig. 1. Ultrasonographic findings of the SCM in a patient with congenital muscular torticollis. (A) Right side and (B) left side. SCM, sternocleidomastoid muscle.

form an electrophysiological examination due to the caregiver's refusal. As a treatment, she received physical therapy including manual neck stretching exercise and active-assisted and passive ROM exercise. In the supine position, affected SCM stretching was achieved by hyperextension of the neck and rotation of the head. The follow-up after 1 month showed a positive outcome. Additionally, home exercise was performed by parents for another 1 month. After that, her head and chin were in a central position and showed symmetrical rotation with no restriction to flexion or extension of the cervical spine. The ROM in the neck was flexion $35^{\circ}$, extension $30^{\circ}$, lateral flexion right/left $25^{\circ} / 20^{\circ}$, and rotation right $/$ left $80^{\circ} / 60^{\circ}$. She looked comfortable when rotating her neck to bilateral sides.

\section{DISCUSSION}

Congenital muscular torticollis is a neck deformity primarily involving the shortening of SCM which is detected at birth or shortly after birth. It causes ipsilateral tilt of the head and contralateral rotation of the face, chin, and facial asymmetry. Craniofacial asymmetry is a coexisting impairment in up to $90 \%$ of infants with congenital muscular torticollis [4]. It may be accompanied by other neurological or musculoskeletal conditions. Its pathogenesis remains controversial. A number of theories have explained the cause of fibrosis. The theories include intrauterine mechanical factors, birth trauma (including use of instruments for delivery), infection, and prenatal or perinatal compartment syndrome. Congenital muscular torticollis is typically categorized as three types: postural, muscular, and SCM mass congenital muscular torticollis [5]. First, postural congenital muscular torticollis presents as the infant's postural preference to one side, without passive ROM restriction, and it is the mildest form. Second, muscular congenital muscular torticollis presents with SCM tightness and passive ROM limitation. Third, infants with an SCM mass present with a fibrotic thickening of the SCM and passive ROM limitation, which is the most severe form. In general, infants with postural congenital muscular torticollis take a short period of treatment, but infants with SCM mass take a longer period of treatment [6].

Several physical examinations can be performed on infants with suspected or diagnosed congenital muscular torticollis including bilateral passive/active ROM of cervical rotation and lateral flexion; passive/active ROM of the trunk; screening for developmental dysplasia of the hip; pain or discomfort at rest; during passive/active movement, skin integrity of neck and presence of SCM mass; craniofacial asymmetries; and head shape [5]. Ultrasound imaging can quantify the shape, size, and location of fibrous mass of SCM. Early treatment is associated with an increase in treatment effectiveness, a significant reduction in SCM thickness, and a reduction in the need for surgical intervention.

Physical therapy management of congenital muscular torticollis is comprehensive, not just stretching neck muscles. Comprehensive care includes positioning, environmental adaptations, passive 
stretching to elongate shortened SCM, and parent/caregiver education. In the case of refractory torticollis, surgical intervention could be a choice [5].

Congenital muscular torticollis is known to be associated with deformational plagiocephaly, developmental dysplasia of the hip, and brachial plexus injury which was suspicious of Erb-Duchenne palsy in this case. It has yet to be addressed in the medical literature how congenital muscular torticollis and brachial plexus injury affects each other. Hervey-Jumper et al. [7] have reported the incidence of perinatal torticollis occurring concurrently with neonatal brachial plexus injury was $43 \%$ in the single-institution retrospective review. Thus, early diagnosis and intervention are crucial for the treatment and prevention of associated complications.

We report a case of bilateral congenital muscular torticollis who underwent physical therapy with home exercise. Bilateral torticollis is a very rare form of congenital torticollis. There have been just a few cases reported for bilateral congenital muscular torticollis in the medical literature. Matuszewski et al. [8] have reported a case of a boy with bilateral congenital torticollis and 25 years of followup, who was diagnosed at the age of 12 , had tenotomies on both SCM and had a full recovery after 6 months, with no restriction to all ROM after 25 years. Babu et al. [9] have reported a case of a girl with bilateral congenital torticollis, who was diagnosed at the age of 19, had bilateral unipolar inferior SCM resection, and got a better ROM of the neck after 3 months. Through physical therapy including passive and active ROM exercises in the hospital by a physical therapist and at home by parents, this patient showed great outcome and had motor recovery of the right upper extremity from grade 1 to 5 gradually. Therefore, we should take into careful consideration the possibility of brachial plexus injury, extremity deformities, developmental delays, and facial asymmetry. Thus, early diagnosis and treatment are crucial for secondary or concomitant deficits and the prevention of future complications.

\section{REFERENCES}

1. Chen MM, Chang HC, Hsieh CF, Yen MF, Chen TH. Predictive model for congenital muscular torticollis: analysis of 1021 infants with sonography. Arch Phys Med Rehabil 2005;86:2199-203.

2. Cheng JC, Au AW. Infantile torticollis: a review of 624 cases. J Pediatr Orthop 1994;14:802-8.

3. Tom LW, Rossiter JL, Sutton LN, Davidson RS, Potsic WP. Torticollis in children. Otolaryngol Head Neck Surg 1991;105:1-5.

4. Cheng JC, Tang SP, Chen TM, Wong MW, Wong EM. The clinical presentation and outcome of treatment of congenital muscular torticollis in infants: a study of 1,086 cases. J Pediatr Surg 2000;35:1091-6.

5. Kaplan SL, Coulter C, Sargent B. Physical therapy management of congenital muscular torticollis: a 2018 evidence-based clinical practice guideline from the APTA academy of pediatric physical therapy. Pediatr Phys Ther 2018;30:240-90.

6. Celayir AC. Congenital muscular torticollis: early and intensive treatment is critical: a prospective study. Pediatr Int 2000;42:504-7.

7. Hervey-Jumper SL, Justice D, Vanaman MM, Nelson VS, Yang LJ. Torticollis associated with neonatal brachial plexus palsy. Pediatr Neurol 2011;45:305-10.

8. Matuszewski L, Pietrzyk D, Kandzierski G, Wilczynski M. Bilateral congenital torticollis: a case report with 25 years of follow-up. J Pediatr Orthop B 2017;26:585-8.

9. Babu MK, Lee P, Mahadev A, Lee EH. Congenital bilateral sternocleidomastoid contracture: a case report. J Pediatr Orthop B 2009;18:145-7. 